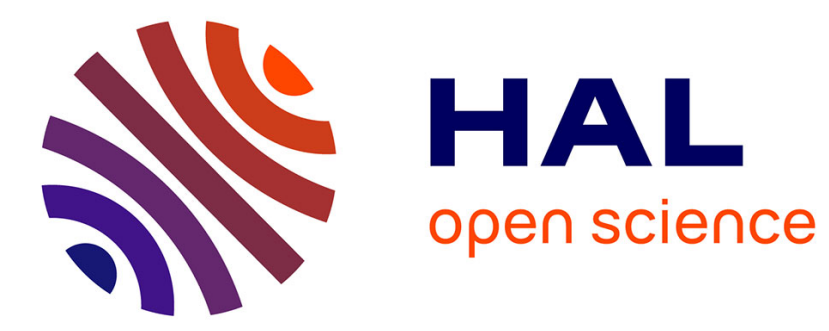

\title{
Radiation belt activity indices and solar proton event alarm on the craterre project web site
}

D. Lazaro, D. Boscher, S. Bourdarie, A. Sicard-Piet, G. Rolland, R. Ecoffet, E. Lorfèvre

\section{- To cite this version:}

D. Lazaro, D. Boscher, S. Bourdarie, A. Sicard-Piet, G. Rolland, et al.. Radiation belt activity indices and solar proton event alarm on the craterre project web site. Radiation Effects on Components and Systems 2013 RADECS 2013, Sep 2013, OXFORD, United Kingdom. hal-01057374

HAL Id: hal-01057374

https: / hal-onera.archives-ouvertes.fr/hal-01057374

Submitted on 22 Aug 2014

HAL is a multi-disciplinary open access archive for the deposit and dissemination of scientific research documents, whether they are published or not. The documents may come from teaching and research institutions in France or abroad, or from public or private research centers.
L'archive ouverte pluridisciplinaire HAL, est destinée au dépôt et à la diffusion de documents scientifiques de niveau recherche, publiés ou non, émanant des établissements d'enseignement et de recherche français ou étrangers, des laboratoires publics ou privés. 


\title{
Radiation Belt Activity Indices and Solar Proton Event Alarm on the CRATERRE Project Web Site
}

\author{
D. Lazaro, D.Boscher, S.Bourdarie, A.Sicard-Piet, G. Rolland, R. Ecoffet, E. Lorfèvre
}

\begin{abstract}
Two Radiation Belt Activity Indices, based on electron flux measurement $>300 \mathrm{keV}$ and $>1.6 \mathrm{MeV}$, and one Solar Proton Event Alarm, based on proton flux measurement $>75 \mathrm{MeV}$, are developed for post events analysis. Both indices and alarm are plotted over the last 30 days on the CRATERRE project web site and are daily updated.
\end{abstract}

Index Terms - CRATERRE project, Radiation belts activity, Space environment

\section{INTRODUCTION}

$\mathbf{S}_{\mathrm{r}}^{\mathrm{p}}$ PACECRAFT engineers, operators and radiation belts researchers share a common desire to understand and predict the structure and variability of Earth's radiation belts. In the radiation belts community, there is a need for improved scientific understanding of the radiation belts, more accurate dynamic and climatology models, space weather restitution and prediction and a mechanism for more efficient transfer of scientific comprehension to the space community.

To allow for such advancements to take place, the CRATERRE project (Ceintures de RAdiation de la TERRE ) [1] aims to organize studies around environment specification models of the radiation belts. In the frame of this project, a web virtual observatory, IPSAT (Ionizing Particle in Space Analysis Tool) [2] has been developed to analyze and visualize in-situ measurements gathered in a local database IPODE (Ionizing Particle Onera DatabasE).

In the present paper, two aspects of those activities are highlighted. Firstly the CRATERRE project is presented in details and it is explained how, in this framework, data measurements are continuously collected. Secondly, some of those data are daily extracted from IPODE, exploited and formatted to provide, as a space weather service, friendly and easy to understand information about the Earth radiation belts activity for post event analysis.

The advantage of these new alerts over more complex existing ones, is to give, at one glance, light but reliable information. The global methodology to determine those indices is to select a sensor channel, along a dedicated orbit, with an energy range representative of the induced effects. Those channel measurements are extracted and a spatial criteria is applied to determine the activity region of interest. Resulting filtered fluxes are compared to threshold levels to produce a color code warning. Those threshold levels reflecting space environment activity are adjusted thanks to

\footnotetext{
The research leading to these results has received funding from CNES under contract W-ECTP/04-06-01.

D.Lazaro, D.Boscher, S.Bourdarie, A.Sicard-Piet are with ONERA - The French Aerospace Lab, F-31055, Toulouse, France (e-mail: didier.lazaro@onera.fr).

G.Rolland, R.Ecoffet and E.Lorfèvre are with CNES, 18 av. E. Belin 31401 Toulouse, France(email : Guy.Rolland@cnes.fr)
}

expertise of data collected for years and based on cumulative distribution function. Then, two Radiation Belt Activity Indices and one Solar Proton Event Alarm are developed from measurements of sensor channels aboard METOPA/SEM2 and JASON-2/ICARENG. Those monitors are of particular interest to study the ionizing radiation inducing damage in electronic boxes.

\section{THE CRATERRE PROJECT}

The CRATERRE project, developed at ONERA was created in 2005 and funded by CNES, the French Space Agency. This project is still going on and is divided into three tasks:

- Supplying and analyzing in-flight measurements,

- Radiation belts physical modeling improvement,

- Radiation belts specification models development.

Regarding in-flight measurements, one outcome of this project has been to write a standard procedure for analyzing measurements in terms of contamination, saturation, background noise, bad data, lack of data and for validating detector measurements with a level of quality as good as possible at the time of analysis. This procedure has been proposed and adopted in the framework of COSPAR/PRBEM (COmmittee on SPAce Research : Panel for Radiation Belt Environment Modeling) [3].

A second outcome has been to standardize measurements database by defining a common file format : CDF (Common Data Format) and its rules of creating and writing it. Raw data are generally in various file format (ASCII, binary, CDF, HDF, NetCDF, CSV , ...), described with different time bases and ephemeris in different system coordinates. Those data are then converted into CDF standard file format, with same time format dependence and unique system of geographic and geomagnetic coordinates. Under the umbrella of the COSPAR/PRBEM, as an international collaborative effort, a dedicated set of codes has been settled and shared into the IRBEM library (International Radiation Belt Environment Modeling) dedicated to radiation belts modeling [4] allowing thus data analyse, inter-calibration, interpolation, exchange.

This procedure led to establish the experimental database named IPODE. This database contains data from many radiation detectors operating on many spacecraft or platforms (France, USA, Russia, Japan, ESA, Argentina, etc.). This is a unique concentration of data, available for the understanding and modeling of the Earth's radiation environment. Measurements have been and are still collected through open source data or collaborations between ONERA and international institutions (Fig. 1). Most world-wide missions having (or having had) radiation monitors on board are gathered here. IPODE is composed of nearly a hundred of spacecraft/instrument couples for in situ Earth's ionising 
particle measurements (electron, proton and alpha particles fluxes) and even heavy ions in the interplanetary medium. The energy range covers roughly $\sim 1 \mathrm{keV}$ to few $\mathrm{MeV}$ for electrons, $\sim 1 \mathrm{keV}$ to few $100 \mathrm{MeV}$ for protons. A large range of orbits are covered from LEO to HEO and GTO and are represented in Fig. 2. The data time resolution ranges for few seconds to minutes depend on the mission itself.

Finally, to be able to visualize and analyze the in-situ measurements provided by IPODE, a dedicated tool named IPSAT has been developed and is used internally at ONERA. IPODE feeds continuously IPSAT with a permanent update, as soon as new raw data are available, relying on a set of bash jobs managed by a Linux crontab. A web version of this virtual observatory is also available for external users, subject to access rights given by PI of instruments. This web tool is accessible through the CRATERRE web site after registration. It offers access to near real time measurements and historical data, by providing an easy and friendly to use interface, to analyse and visualize measurements, locate in flight anomaly with in situ environment from various sources.

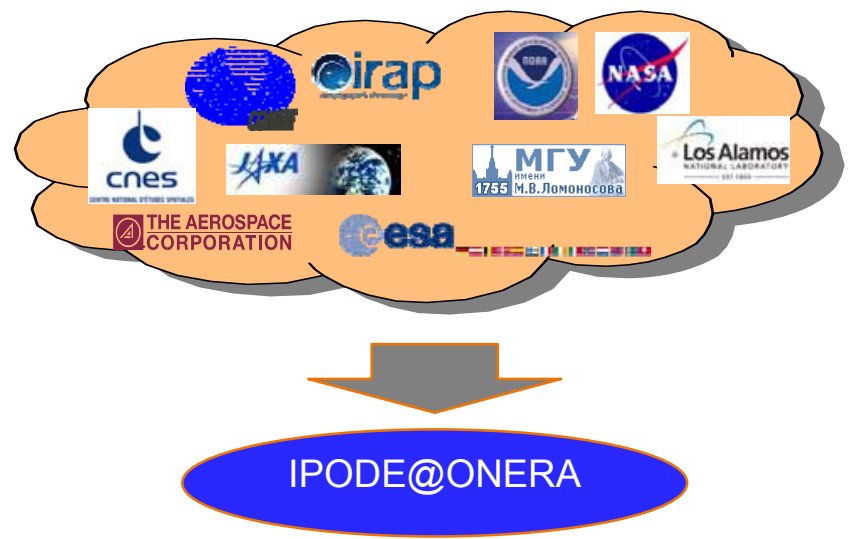

Fig. 1. International collaboration between ONERA and international institutions under the umbrella of the CRATERRE Project

After several years, the CRATERRE project has led to significant advances in radiation belts detection and comprehension with in-situ measurement analysis aboard the French Spacecraft JASON-2 [5], physical modeling improvement with for example electron radiation belt data assimilation [6] and specification models development with new models of electron fluxes such as OZONE in the outer zone electron belt [7], model at MEO orbit (MEO V2 [8]), at geostationary orbit (IGE 2006 [9]) and in the slot region [10].

On the CRATERRE web site, the objectives of the project are summed up, a link to IPSAT is provided and a description of CNES-ONERA ICARE radiation monitor is given. Recently on the homepage, an illustration of IPODE exploitation with IPSAT is made to provide an overview of last month space environment. Daily updated plots present solar protons measured by GOES spacecraft, Kp magnetic activity index and $\mathrm{L}$ versus time plot along JASON-2 orbit for ICARENG measurements : electrons (differential omnidirectional flux at $1.6 \mathrm{MeV}$ ) and protons (integral omnidirectional flux $>97 \mathrm{MeV}$ and differential omni-directional flux at $27 \mathrm{MeV}$ ). Indices of space environment, which are described in this present paper, are also presented with solar proton event alarm based on METOP-A measurements, radiation belt index in the outer region for medium energy electron $(>300 \mathrm{keV})$ with METOP-A measurements and for high energy electron $(>1.6 \mathrm{MeV})$ with JASON-2 measurements.

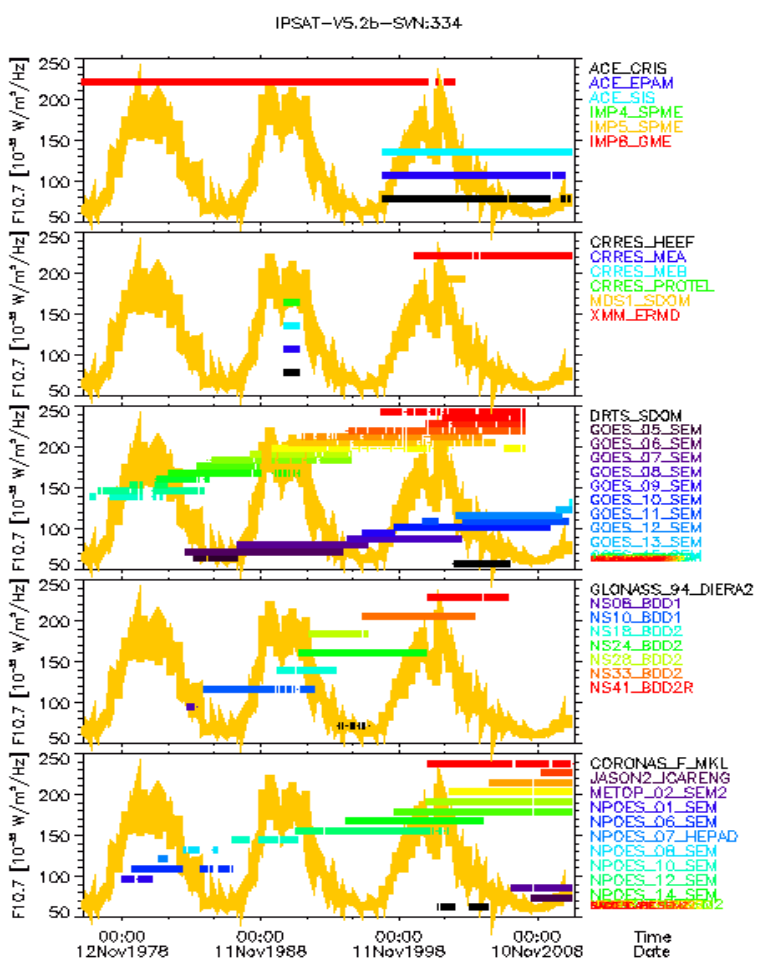

Fig. 2. Time coverage of available spacecraft/instrument couples in IPODE is plotted over the forty last years represented by the $10.7 \mathrm{~cm}$ radio flux of three last solar cycles (yellow plot). From top to bottom panels, missions and orbits are plotted : 1-Interplanetary missions, 2-HEO-GTO, 3-GEO, 4-MEO, 5-LEO-PEO orbits.

\section{RADIATION BELT ACTIVITY INDICES}

For radiation belt activity indices, some products already exist as for example those provided by the Space Weather Prediction Center at NOAA [11] based on NOAA POES spacecraft measurements (National Polar-orbiting Operational Environmental Spacecraft). NPOES spacecraft are nowadays a constellation of 5 meteorological spacecraft on a $850 \mathrm{~km}$ polar orbit, with second generation Space Environment Monitor SEM-2 data since July, 1998. To discriminate between regions of different geomagnetic particle trapping, three separate belt indices are calculated based on the L-value at the spacecraft's location. The inner zone, the slot region and the outer zone correspond respectively to region with an L-value less than 2.0, region from 2.0 to 2.5 , and region greater than 2.5. The display, proposed on the website, shows the responses of the $90^{\circ}$ electron sensor to $>30 \mathrm{keV}$ electrons throughout a recent day compared with the median responses of that detector over the past year. The $90^{\circ},>30 \mathrm{keV}$ electron sensor is one of 22 channels in SEM-2 detector. For the three regions previously defined, ratio are given ranging from less than 1.0 (the particle intensities are below the median signifying low or very low fluxes compared to the one-year median value or at 
back-ground level) to larger than 1.0 (the particle intensities are higher than the median signifying intense particle fluxes). The value of the ratio is color coded and plotted at the geographic location of the measurement. Ratios near 1.0 signify nominal conditions.

\section{A. $300 \mathrm{keV}$ radiation belt index}

In the same spirit, the idea has been to determine an index based on a dataset that globally reflects radiation belt dynamic state as a reference for the outer zone. On one hand, for low energies (typically below $100 \mathrm{keV}$ ) and in the inner belt, electron behaviour is driven mainly by convection so a ranking with $\mathrm{Kp}$ seems to be enough thus a belt index is not necessary. On the other hand, for higher energy (above 100 $\mathrm{keV}$ ) acceleration and radial diffusion drive the electron dynamic so the definition of an index makes sense. That is the reason why a first index is defined at $300 \mathrm{keV}$. Typically at this energy, convection is no more present and most of low altitude spacecraft measure this energy range, in particular NPOES spacecraft for more than one solar cycle and a half.

To define this new index, the response of the $90^{\circ}$, $>300 \mathrm{keV}$ electron sensor aboard NPOES-15 and METOP-A are used. METOP-A is one meteorological spacecraft developed by ESA and EUMETSAT on the same orbit as NPOES constellation since December, 2006 and with same NOAA SEM2 detector on board. Both datasets are daily averaged around $\mathrm{L}=4$ over the spacecraft time period, respectively from July, 1998 to December, 2012 for NPOES-15 and from December, 2006 to December, 2012 for METOP-A. The results are presented in Fig. 3.

First it can be noticed that METOP-A data are overlapped with NPOES-15 data for the same time period, then it is observed a high dynamic behaviour of fluxes according to time. For NPOES-15 dataset, fluxes deeply vary between 1.5 x $10^{+2} \mathrm{~cm}^{-2} \mathrm{~s}^{-1} \mathrm{sr}^{-1}$ and $6.8 \times 10^{+5} \mathrm{~cm}^{-2} \mathrm{~s}^{-1} \mathrm{sr}^{-1}$ with periods of weak fluxes in 1999 and 2004 and periods of intense fluxes in 2003. The average, over the fourteen years period, is around $\mathrm{A}=3.5 \times 10^{+4} \mathrm{~cm}^{-2} \mathrm{~s}^{-1} \mathrm{sr}^{-1}$, giving a ratio 20 between maximum and average values. The minimum phase of the solar cycle is visible from 2007 to 2010 .

A critical point is to determine a reference level in terms of flux value below which the activity is considered as nominal and some flux values steps to turn activity enhancement into significant warnings, taking into account this large dynamic. To achieve such a goal, those criteria are established with a CDF (Cumulative Distribution Function) with the available datasets and three percentile levels, as flux steps, are selected to define four activity phases :

--Quiet phase with fluxes less than the 60-percentile,

--Moderate phase with fluxes between the 60-percentile and the 85-percentile,

--Active phase with fluxes between the 85-percentile and the 98-percentile,

--Extreme phase with fluxes greater than the 98-percentile.

Percentiles, thus defined, give the fraction of points, from the total distribution, which is under the corresponding flux level or another way to say, the percentage of time the daily averaged fluxes are in one class or another one. This CDF is plotted in Fig.4 in blue line for NPOES-15 and in red line for METOP-A. As the METOP-A time coverage is shorter than the NPOES-15 one, and moreover during the minimum phase of the solar cycle, the METOP-A CDF is biased and not enough representative to get a good statistic and define classes. On the other side, NPOES-15 statistic is seen as significant for the settlement of the CDF and to define flux level values corresponding to the selected percentiles. They are reported in Table I.

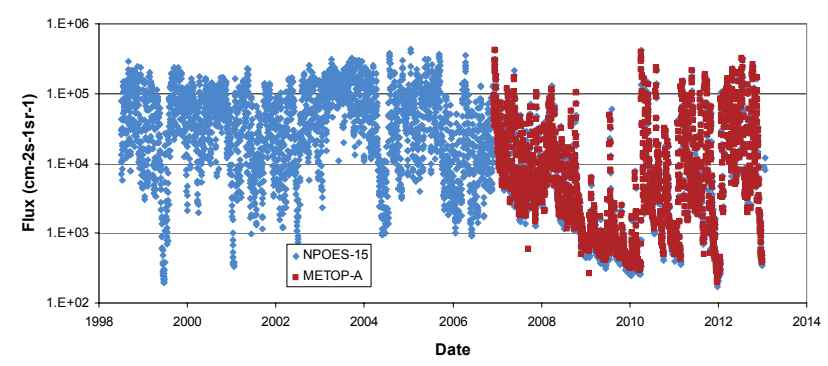

Fig. 3. Daily average of $90^{\circ},>300 \mathrm{keV}$ electron fluxes around $\mathrm{L}=4$ aboard NPOES-15 from July, 1998 to December, 2012 and aboard METOP-A from December, 2006 to December 2012.

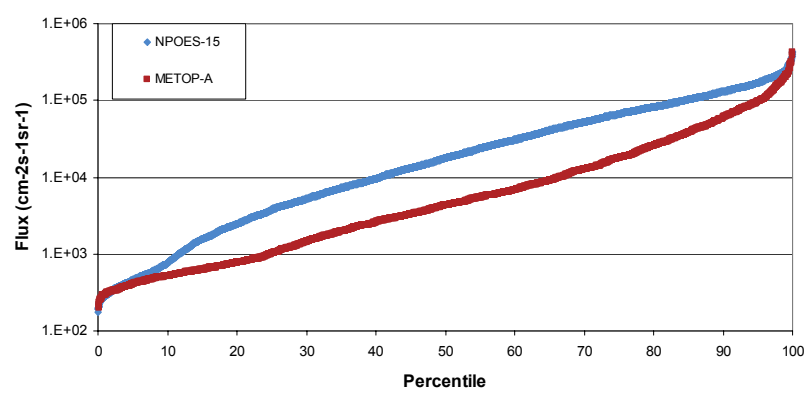

Fig. 4. Cumulative Distribution Function of daily average of $90^{\circ},>300 \mathrm{keV}$ electron fluxes around L=4 aboard NPOES-15 from July, 1998 to December, 2012 and aboard METOP-A from December, 2006 to December 2012.

TABLE I

CLASSIFICATION OF DAILY AVERAGE FLUXES AROUND L $=4$ ABOARD NPOES-15 ACCORDING TO CLASS DEFINITION

\begin{tabular}{|c|c|c|}
\hline \hline Environnement & \% of time & Flux value $\left(\mathrm{cm}^{-2} \mathrm{~s}^{-1} \mathrm{sr}^{-1}\right)$ \\
\hline Quiet & 60.00 & Flux $<3.08 \times 10^{+4}$ \\
\hline Moderate & 25.00 & $3.08 \times 10^{+4}<$ Flux $<1.03 \times 10^{+5}$ \\
\hline Active & 13.00 & $1.03 \times 10^{+5}<$ Flux $<2.18 \times 10^{+5}$ \\
\hline Extreme & 2.00 & $2.18 \times 10^{+5}<$ Flux \\
\hline \hline
\end{tabular}

Even if the choice of the percentiles is arbitrary, this is done in the order to have a judicious distribution of the data in the four corresponding classes. The first chosen percentile ( $60 \%$ of time) is close to the average level so the first class covers fluxes lower than the average. The three highest classes cover $40 \%$ of the remaining time and are unevenly distributed to reflect the fact that stronger are the events and more exceptional they are. Moreover the statistic can be enhanced with same datasets also available nowadays aboard 7 probes (NPOES constellation : 15, 16, 17, 18 \& 19 and METOP-A \& B), eventually allowing to average spatially the data.

This class definition based on a cumulative distribution function has the following interests :

--It takes into account the whole dynamic of the distribution and selecting classes is flux level independent and done on the 
fraction of time. It allows to compare index from one dataset to another one.

--The choice of the number of percentiles and their values (here three with the following values : 60, 85 \& 98) can give the alarm the required sensitivity.

--Using such data formatting is close to the vision of specification models parameterized according to confidence levels.

This index definition, based on NPOES-15 statistic, has been implemented with METOP-A/SEM2 data and is automatically updated as soon as new raw data are available in IPODE. Raw data are processed to extract $90^{\circ}, 300 \mathrm{keV}$ electron channel ; position is estimated with NORAD element thanks to IRBEM Lib and an average level is estimated around $\mathrm{L}=4$ for the last available 24 hours. This new index updates the database which provides an overview of the last month behavior on the CRATERRE web site. A color code is settled : green for quiet phase, yellow for moderate, orange for active and red for extreme phase. An illustration during October, 2012 storm period is presented and commented in paragraph III-C.

\section{B. $\quad 1.6 \mathrm{MeV}$ radiation belt index}

A second radiation belt index is settled with the response of the $>1.6 \mathrm{MeV}$ electron sensor from ICARENG aboard JASON-2. The JASON-2 project is a response to the international demand for programs to study and observe oceans and climate, through a worldwide ocean observation system. JASON-2 is part of a cooperation between CNES, EUMETSAT, NASA and NOAA. Onboard JASON-2, the CARMEN French instrument is included and measures high energy particles with the ICARENG detector [4]. This spacecraft is flying on a Low Earth Orbit (altitude : $1336 \mathrm{~km}$, inclination : $66^{\circ}$ ) since June, 2008.

This new index is defined in the same manner as the first one, but before doing this, it is interesting to evaluate the behavior of fluxes at this energy value compared to the previous one. During the time coverage in common between NPOES-15 and JASON-2 (mid-2008, mid-2012), daily averaged fluxes around $\mathrm{L}=4$ for both energies $(>300 \mathrm{keV}$ and $>1.6 \mathrm{MeV}$ ) are plotted in Fig. 5. A clear correlation is observed between both fluxes evolution however it is not possible to determine a reliable correlation factor. This is due to the fact that the dynamic is not the same between both energy ranges. This can be illustrated in Fig. 6 by plotting the ten highest daily averaged fluxes observed in JASON-2 data distribution for $>1.6 \mathrm{MeV}$ energy and for others channels available with this detector (between $1.6 \mathrm{MeV}$ and $2.6 \mathrm{MeV}$ ) and by plotting the $>300 \mathrm{keV}$ flux level for the same date. The AE8 Min and Max simulated spectra are added for linking both datasets. It is then confirmed the dynamic difference between both energies; this dynamic is more important at $>300 \mathrm{keV}$ energy. At $>1.6 \mathrm{MeV}$, the maximum flux is $1.2 \times 10^{+4} \mathrm{~cm}^{-2} \mathrm{~s}^{-1} \mathrm{sr}^{-1}$ and the average level is $1.0 \times 10^{+3}$ $\mathrm{cm}^{-2} \mathrm{~s}^{-1} \mathrm{sr}^{-1}$, giving a ratio equal to 12 between maximum and average values.

Same CDF is done with $>1.6 \mathrm{MeV}$ dataset and is plotted in Fig.7. Same percentiles are defined and the flux level values corresponding to the selected percentiles are obtained. They are reported in Table II. Obviously, the statistic is rather weak and the measurement points are obtained during the minimum and the rising phase of the solar cycle. Moreover it has to be taken into account this occurs during a particularly weak solar cycle. It induces a CDF right shifted towards high percentile values because the weight of low flux values is more important. One can expect stronger geomagnetic storms with the incoming solar maximum and declining phase and it could be considered within the future months or years if it is necessary to reconsider the CDF and adjust the flux values corresponding to the chosen percentiles.

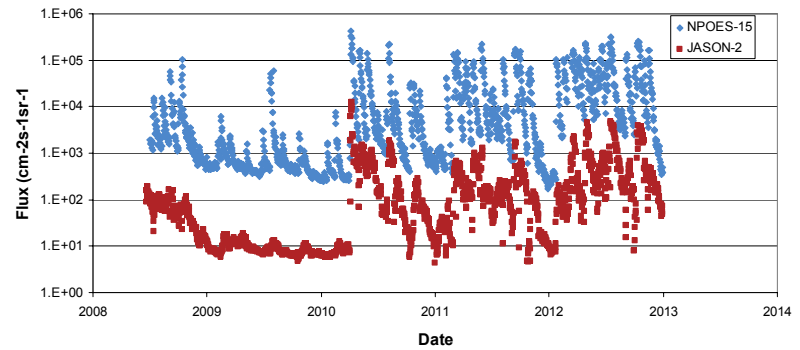

Fig. 5. Daily average around $\mathrm{L}=4$ of $90^{\circ},>300 \mathrm{keV}$ electron fluxes aboard NPOES-15 (blue line) and $>1.6 \mathrm{MeV}$ electron fluxes aboard JASON-2 (red line) from July, 2008 to July, 2012.

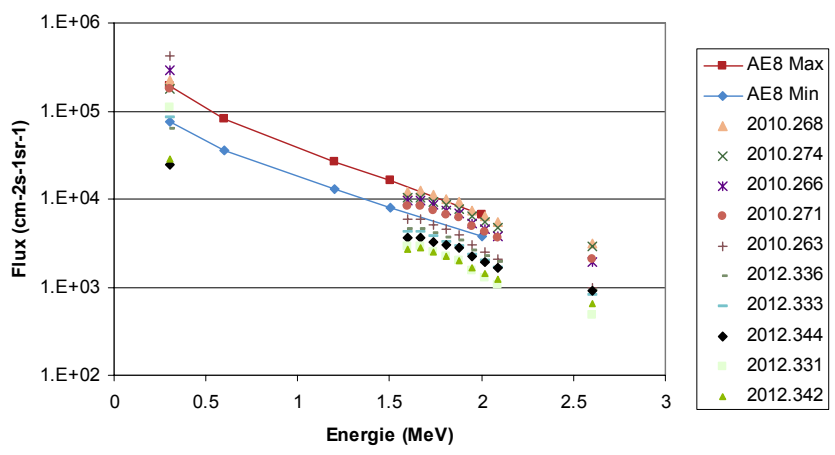

Fig. 6. Representation of the ten highest fluxes vs. energy from distribution plotted in Fig.5 around $>1.6 \mathrm{MeV}$ (JASON-2) and $>300 \mathrm{keV}$ (NPOES-15) for the same date. Comparison is done with AE8 Min and Max spectra.

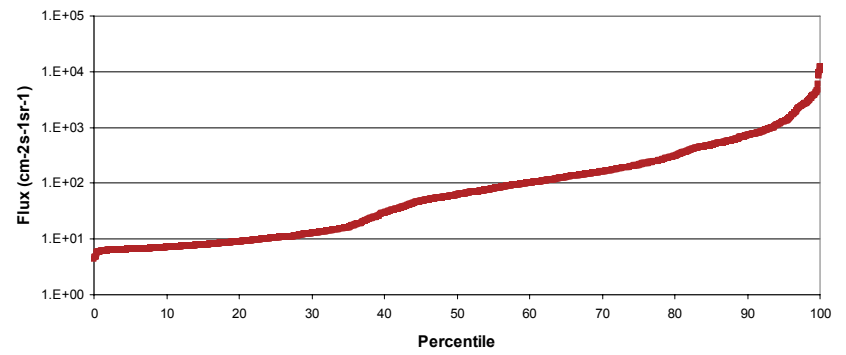

Fig. 7. Cumulative Distribution Function of daily average of $>1.6 \mathrm{MeV}$ electron fluxes around L=4 aboard JASON-2 from July, 2008 to December, 2012

TABLE II

CLASSIFICATION OF DAILY AVERAGE FLUXES AROUND L $=4$ ABOARD JASON-2 ACCORDING TO CLASS DEFINITION

\begin{tabular}{|c|c|c|}
\hline \hline Environnement & \% of time & Flux values $\left(\mathrm{cm}^{-2} \mathrm{~s}^{-1} \mathrm{sr}^{-1}\right)$ \\
\hline Quiet & 60.00 & Flux $<1.01 \times 10^{+2}$ \\
\hline Moderate & 25.00 & $1.01 \times 10^{+2}<$ Flux $<4.80 \times 10^{+2}$ \\
\hline Active & 13.00 & $4.80 \times 10^{+2}<$ Flux $<2.68 \times 10^{+3}$ \\
\hline Extreme & 2.00 & $2.68 \times 10^{+3}<$ Flux \\
\hline \hline
\end{tabular}


The process of this second index is implemented in the same manner as the first one and an update is done as soon as new ICARENG data are available. The same color code is used and an overview of the last month behavior is provided on the CRATERRE web side. As well as for the $>300 \mathrm{keV}$ index, an illustration during October, 2012 period is presented and commented in the next paragraph III-C.

\section{Observations and validation}

During October 2012, a series of geomagnetic perturbations illustrates the behavior of both indices. In Fig.8, the magnetic activity, $\mathrm{Kp}$ index, is plotted in the bottom panel. Then from bottom to top, the panels show the radiation belt index for $1.6 \mathrm{MeV}$, JASON-2 measurements in a $\mathrm{L}^{*}$ versus time plot for $>1.6 \mathrm{MeV}$ electron, the radiation belt index for $>300 \mathrm{keV}$ and finally METOP-A measurements in a $\mathrm{L}^{*}$-versus time plot for $>300 \mathrm{keV}$ electron.

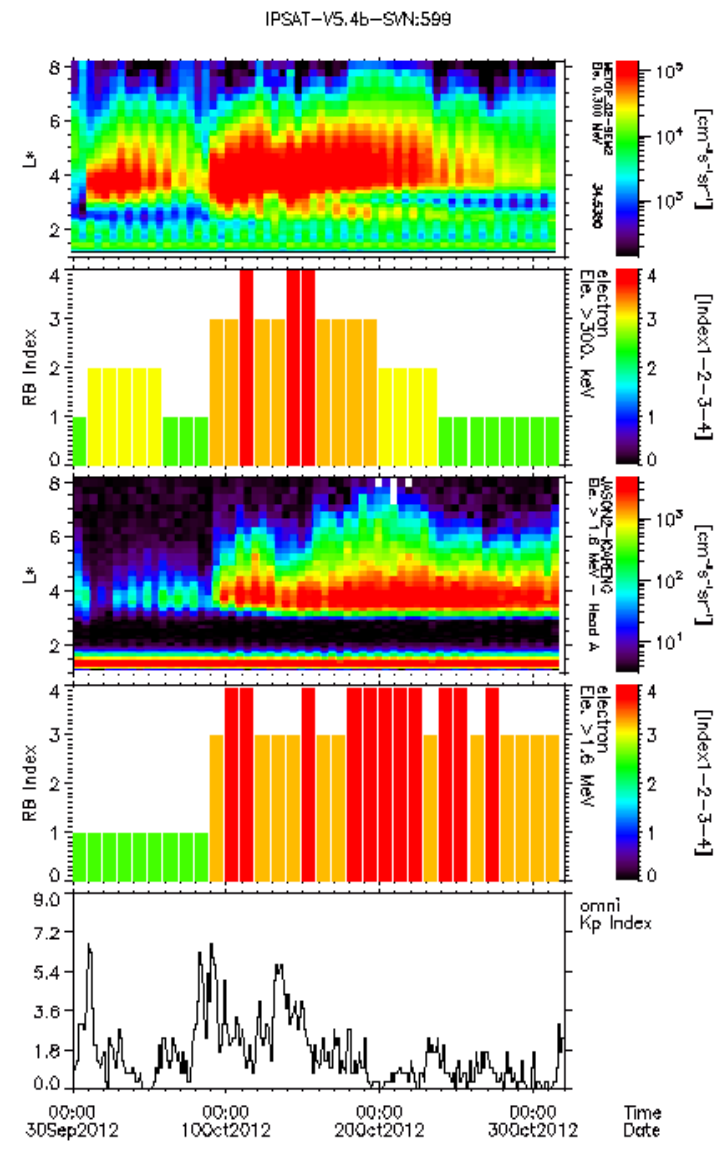

Fig. 8. During October 2012, the magnetic activity, Kp index, is plotted in the bottom panel, then from bottom to top, the panels show the radiation belt index for $1.6 \mathrm{MeV}$, JASON-2 measurements in a $\mathrm{L}^{*}$ versus time plot for $>1.6$ $\mathrm{MeV}$ electron, the radiation belt index for $300 \mathrm{keV}$, METOP-A measurements in a $\mathrm{L}^{*}$-versus time plot for $>300 \mathrm{keV}$ electron

At the beginning of the month a CME (Coronal Mass Ejection) induces a high and brief $\mathrm{K}_{\mathrm{p}}$ increase around 7. It is observed, during the same time an increase of flux along METOP-A orbit and the index, initially green ('Quiet') turns to yellow ('Moderate') during 5 days. Flux at high energy along JASON-2 orbit does not change and the corresponding index remains green. The perturbation is intense but too brief to accelerate electrons to high energy. Then a few days later, it is observed a second increase of $\mathrm{K}_{\mathrm{p}}$ not as high as the first one (around 5) but lasting a few days. Both medium energy and high energy fluxes increase and remain at high level during several days inducing 'Active' and 'Extreme' activities. The medium energy environment returns to 'Quiet' state sooner than the high energy environment which stays 'Active' and 'Extreme' still a few days more. Both behaviors, during the increasing and the decreasing phases of the storm show that the system at high energy is more inertial than the one at low energy. Even if one can think that the high levels of alarm reached by the index at high energy are biased by the weak statistic, this main conclusion about the system inertia, is reinforced by the behavior of the index remaining green during the first part of the storm.

\section{SOLAR PROTON EVENT ALARM}

\section{A. Alarm definition}

An alarm for Solar Proton Event (SPE) Hazard is provided based on SEM2 proton measurements aboard NPOES-15 and METOP-A (same raw data as for radiation belt indices). The methodology of the study is the following : the SPE hazard condition is based on one SEM2 proton channel data $(>75 \mathrm{MeV})$ because protons with such energy are likely to induce single event effect on board electronics. A spatial criteria based on McIlwain $\mathrm{L}_{\mathrm{m}}$ parameter calculation with geographic coordinates as input is necessary to determine when the spacecraft is inside or outside trapped radiation belt. $\mathrm{L}_{\mathrm{m}}$ is chosen as spatial criteria rather than $\mathrm{L}^{*}$ because $\mathrm{L}_{\mathrm{m}}$ is best suitable to define open-close field lines and therefore regions where solar flare particles can have access to ; otherwise $\mathrm{L}^{*}$ allows to define trapping regions only.

To illustrate the determination of the SPE hazard condition, proton flux from $>75 \mathrm{MeV}$ channel and $\mathrm{L}_{\mathrm{m}}$ versus time for NPOES-15 during a few hours are plotted in Fig.9. The left side of the plot presents a time period without SPE ; every orbital period ( 101 minutes), the spacecraft crosses the proton radiation belt when $\mathrm{L}_{\mathrm{m}}$ values are typically less than 2.5 or 3 ; at the same time a peak flux is observed due to radiation belt trapped protons. Outside proton radiation belt, flux levels are low and close to instrument background. The right side of the plot presents a time period during SPE; peak fluxes due to the radiation belt crossing are still observed at low $\mathrm{L}_{\mathrm{m}}$ values.

When the spacecraft is at high $\mathrm{L}_{\mathrm{m}}$ values, at the same time, peak fluxes outside radiation belt due to SPE fluxes are observed. It is then possible to discriminate peak fluxes due to SPE when the spacecraft is outside radiation belt and peak fluxes due to trapped proton when the spacecraft is inside the radiation belt, selecting at the same time the two following conditions to sort the data: proton channel $>75 \mathrm{MeV}$ fluxes greater than instrument background noise and geomagnetic coordinate $\mathrm{L}_{\mathrm{m}}$ greater than predefined value $>2.7$. This geomagnetic coordinate condition is indicated by a black horizontal line at $\mathrm{L}_{\mathrm{m}}=2.7$ on top panels of Fig. 9 . 


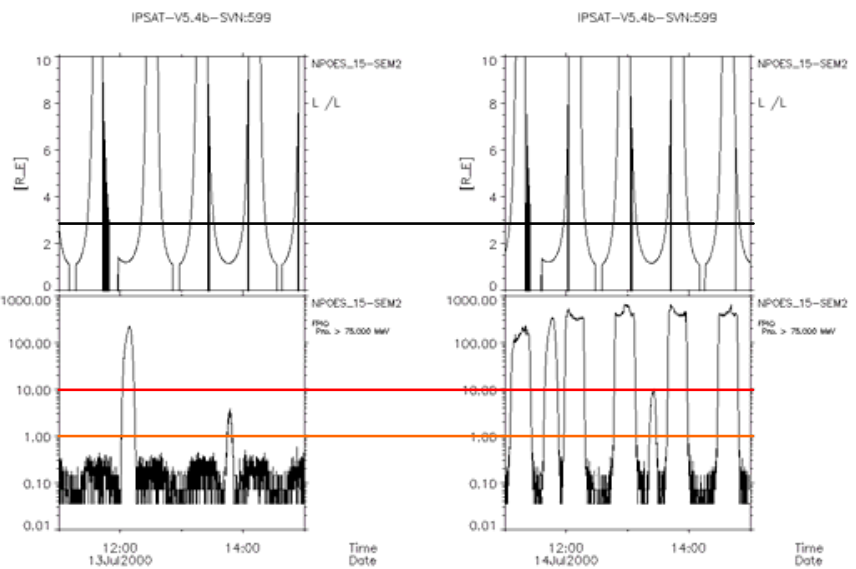

Fig. 9. $\mathrm{L}_{\mathrm{m}}$ (top panels) and $>75 \mathrm{MeV}$ proton channel (bottom panels) versus time; on both left panels : without SPE, only are visible two peak fluxes due to radiation belt and corresponding to low $\mathrm{L}_{\mathrm{m}}$ values ; on both right panels : with SPE, both are visible : two peak fluxes due to radiation belt and five SPE peak fluxes. On top panels, black horizontal line at $\mathrm{L}_{\mathrm{m}}=2.7$ indicates the limit of the radiation belt. On bottom panel, orange and red horizontal lines indicate the position of the two thresholds.

Based on the previous criteria definition, it is proposed to sort the data for a given sampling time range corresponding to the spacecraft orbital period: 101 minutes. Concerning flux level, two thresholds are chosen so three conditions, using a colour code, are defined to modulate the alarm level :

--Quiet condition when flux is below $1 \mathrm{~cm}^{-2} \mathrm{~s}^{-1} \mathrm{sr}^{-1}$ (green color)

--Active condition when flux is over $1 \mathrm{~cm}^{-2} \mathrm{~s}^{-1} \mathrm{sr}^{-1}$ and below $10 \mathrm{~cm}^{-2} \mathrm{~s}^{-1} \mathrm{sr}^{-1}$ (orange color) color)

--Extreme condition when flux is over $10 \mathrm{~cm}^{-2} \mathrm{~s}^{-1} \mathrm{sr}^{-1}$ (red

The two thresholds are represented on Fig. 9 with orange and red lines on bottom panels. Applying this threshold definition, it is possible to know when, at the same time, $\mathrm{L}_{\mathrm{m}}$ is greater than 2.7 and flux meets one of the three level conditions. Then this requirement gives the information if the spacecraft is exposed or not to solar proton flux levels.

\section{B. Validation}

This alarm is validated by scanning of the entire NPOES-15 data available on IPODE. At this time, this spacecraft data base remains the most interesting in the NPOES constellation because it presents the longest life time coverage since July, 1998 until nowadays, that is to say during the entire $23^{\text {rd }}$ solar cycle and the beginning of the present solar cycle. As an example of validation it is presented here the well-known Bastille Day Event occurring July $14^{\text {th }}, 2000$. Top panel of Fig. 10 presents a plot of proton flux channel $>75 \mathrm{MeV}$ seen by NPOES-15 as a function of time during this event. It can easily be seen the recurrent peak flux modulation by the radiation belt and the imprint of the SPE. Middle panel of the figure presents the response of the alarm. This particularly large event generates a red alarm as soon as the flux level increases over the $10 \mathrm{~cm}^{-2} \mathrm{~s}^{-1} \mathrm{sr}^{-1}$ threshold. This red alarms remains more than 30 hours. Then the flux level decreases and generates an orange alarm level during about 24 hours. Bottom panel presents $40-80 \mathrm{MeV}$ flux level observed simultaneously aboard GOES-10 at geostationary orbit. This result illustrates that this alarm defined with LEO data is available and useful whatever the orbit of interest.

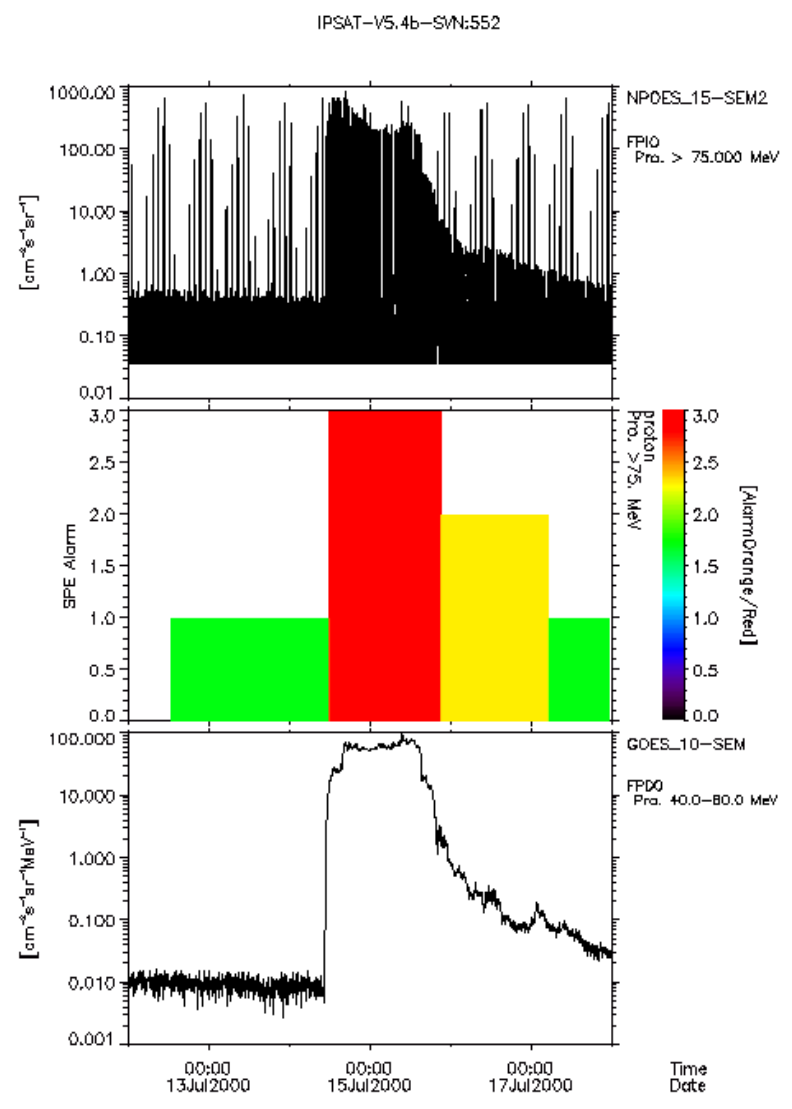

Fig. 10. Analysis of Bastille Day Event with NPOES-15 $>75 \mathrm{MeV}$ flux (top panel), alarm deduced from those data (middle panel) and GOES-10 40-80 $\mathrm{MeV}$ proton flux (bottom panel)

\section{CONCLUSION}

Goal of the ONERA-CNES CRATERRE project is to develop new radiation belt models allowing a better description of the space environment (electron and proton) for mission specifications. To reach this goal, research activities concentrate on physical modeling, specification model development and in-situ measurements. Thanks to IPSAT tool and IPODE database, radiation measurements from world-wide missions are gathered in a standardized format and sanitized with an analyzing procedure to produce highest quality dataset.

Among those datasets continuously updated, measurements collected along METOP-A and JASON-2 orbits are of prime interest to develop two dynamics radiation belt activity indices at medium $(>300 \mathrm{keV})$ and high $(>1.6 \mathrm{MeV})$ energy to characterize outer electron belt and one solar proton event alarm at $>75 \mathrm{MeV}$.

These indices for trapped electrons are defined with the same principle: among the daily data flows, a significant value is extracted (presently the averaged value at $\mathrm{L}=4$ for a given energy range) and compared among the distribution of previous dataset with percentile values. Both indices are 
comparable in the same way and present the advantage of integrating the dynamic behavior. Those indices in the form of color warnings are helpful to understand dynamic behavior of radiation belt during geomagnetic storm. The advantage of such a definition is that it allows to establish, in the same way, various indices based on other dataset with same percentile values and to compare them. The solar proton event alarm also gives a color warning when untrapped protons coming from the sun are impinging spacecraft structure. Those indices are available on the CRATERRE web site and updated actually once a day with a delay between 24 and 48 hours depending on data providers.

One improvement will be to reduce this gap with near real time, by processing new raw data as soon as they are available and by updating web site plots. It could also be interesting to correlate observed events with anomalies data base. Another improvement will be to define new electron indices in the same way, first at low energy for charging effects at geostationary orbit, secondly at very high energy when it occurs during violent geomagnetic, thereby providing a rough outline of an environment activity spectrum.

\section{ACKNOWLEDGMENT}

Authors thank the PIs of all instruments whose data are used in this study and also particularly thank EUMETSAT for the METOP-A data provision.

\section{REFERENCES}

[1] The ONERA-CNES CRATERRE project web site : http://craterre.onecert.fr//home.html.

[2] Bourdarie S., A. Sicard-Piet, D. Boscher, D. Lazaro, R. Ecoffet, G. Rolland and M. Dinguirard, IPSAT : Ionizing Particle in Space Analysis Tool, Acta Astronautica, 63, pp. 471-474 (2008)

[3] http://craterre.onecert.fr//prbem/home.html.

[4] http://irbem.svn.sourceforge.net/viewvc/irbem/trunk/manual/user_guid e.html

[5] Boscher D., Bourdarie S., Falguere D., Lazaro D., Bourdoux P., Baldran T., Rolland G., Lorfevre E., Ecoffet R., In Flight Measurements of Radiation Environment on Board the French Spacecraft JASON-2, IEEE Trans. on Nuclear Science, Vol.58, Issue 3, 916-922, June 2011.

[6] Bourdarie S., Maget V., Electron radiation belt data assimilation with an ensemble Kalman filter relying on the Salammbô code, Ann. Geophys. 30, 929-943, 2012.

[7] Bourdarie S., Sicard-Piet A., Friedel R., O'Brien P., Cayton T., Blake B., Boscher D., Lazaro D., Outer Electron Belt Specification Model, IEEE Trans. on Nuclear Science, Vol.56, Issue 4, 2251-2257, Aug. 2009

[8] Sicard-Piet A., Bourdarie S., Boscher D., Friedel R., Cayton T., Solar cycle electron environment at GNSS like altitudes, IAC-06-D5.2.04, 2006

[9] Sicard-Piet A., Bourdarie S., Boscher D., Friedel R., A model for the geostationary electron environment: POLE, from $30 \mathrm{keV}$ to $5.2 \mathrm{MeV}$, IEEE Trans. Nuc. Sci. 53 (4), 1844-1850, August 2006

[10] Sicard-Piet A., Boscher D., Lazaro D., Bourdarie S., Rolland G., A new ONERA-CNES Slot Electron model, Radecs 2013

[11] Space Weather Now at Space Weather Prediction Center (NOAA) http://www.swpc.noaa.gov/SWN/index.html 